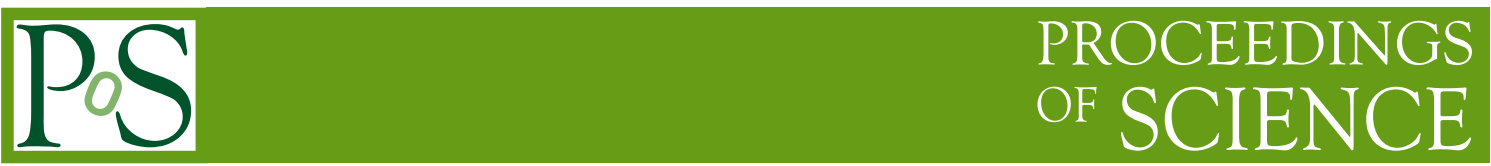

\title{
D* in diffraction at HERA
}

\author{
Roger Wolf ${ }^{* *}$ \\ University of Heidelberg, Germany \\ E-mail: roger.wolfedesy.de
}

Diffractive open-charm production apart from diffractive dijet production provides an independent way to test QCD factorization in diffraction. In this paper recent results for diffractive $D^{*}$ production at the HERA experiments H1 and ZEUS are summarized. They are based on data collected during the years 1998-2000 and cover the kinematic regimes of deep inelastic scattering and photoproduction.

International Europhysics Conference on High Energy Physics

July 21st - 27th 2005

Lisboa, Portugal

* Speaker.

†ON BEHALF OF THE H1 \& ZEUS COLLABORATION. 


\section{Introduction}

QCD, the theory of strong interactions, predicts that the cross section for diffractive deepinelastic electron-proton scattering (DIS) factorizes into a set of universal diffractive parton density functions (DPDFs) of the proton and process-dependent hard scattering coefficients [1]. Next-toleading order (NLO) DPDFs have been determined from QCD fits to the measured reduced cross sections of inclusive diffractive scattering at HERA [3] [4], using the evolution equations by [2] (DGLAP). Those have been found to be dominated by the gluon, which carries $\approx 75 \%$ of the momentum of the diffractive exchange. Open-charm production at HERA is expected mainly to proceed via the Boson Gluon Fusion process as depicted in Fig. 1 and thus is directly sensitive to the gluon content of the diffractive exchange. There a photon of virtuality $Q^{2}$ undergoes a hard

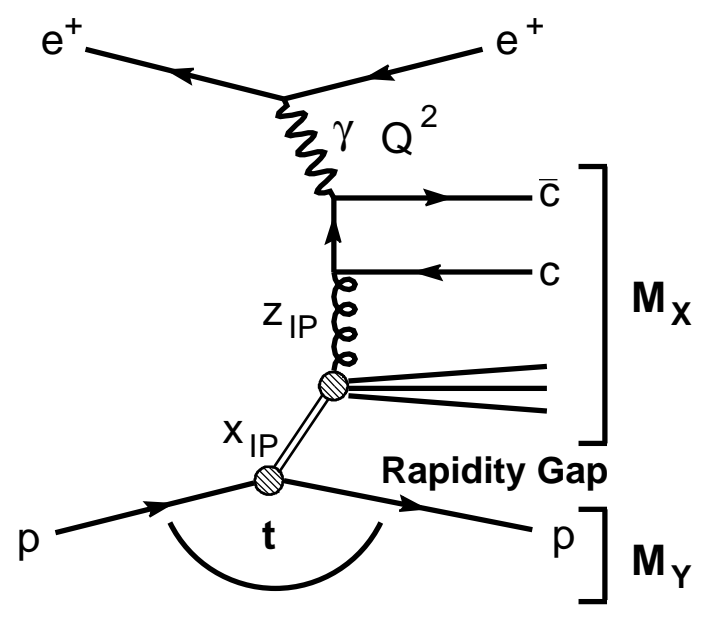

Figure 1: The dominant process of diffractive open-charm production ep $\rightarrow e X Y \rightarrow e\left(D^{*} X^{\prime}\right) Y$ is Boson Gluon Fusion. The electron/positron (e) couples to a photon $(\gamma)$ which interacts with the proton $(p)$ via a diffractive (color singlet) exchange and thus produces two distinct final state hadronic systems $X$ and $Y$, which are separated by the largest gap in rapidity between the final state hadrons.

scatter with a gluon that emerges from the diffractive exchange forming a cic-pair. The diffractive exchange is characterized by a large rapidity gap, which separates two well distinguished hadronic systems, a low mass diffractive system $Y$ in the direction of the outgoing proton and a photon dissociative system $X$ which is experimentally selected to be well confined within the detector volume. The diffractive kinematic variables are defined as

$$
\mathrm{x}_{\mathbf{P}}=\frac{\mathrm{q} \cdot\left(\mathrm{P}-\mathrm{p}_{\mathrm{Y}}\right)}{\mathrm{q} \cdot \mathrm{P}} ; \quad \mathrm{t}=\left(\mathrm{P}-\mathrm{p}_{\mathrm{Y}}\right)^{2} ; \quad \beta=\frac{\mathrm{Q}^{2}}{2 \mathrm{q}\left(\mathrm{p}-\mathrm{p}_{\mathrm{Y}}\right)}
$$

Where $P(q)$ are the 4-momenta of the proton (photon) respectively and $p_{\mathrm{Y}}$ denotes the 4-momentum of system $Y$. The square of the transferred 4-momentum at the proton vertex is indicated as $t, \mathbf{x}_{\mathbf{P}}$ is the fractional proton momentum carried by the diffractive exchange and $\beta$ is the fractional momentum of the diffractive exchange carried by the struck quark. If factorization is applicable for diffractive open-charm production, QCD calculations based on DPDFs extracted from inclusive diffractive measurements should be capable to predict diffractive $D^{*}$-meson production in shape and normalization. 
One published measurement in DIS from ZEUS [5] and two further preliminary analyses from ZEUS [6] and H1 [7] are presented, the latter being perfomred in DIS (H1) and photoproduction (ZEUS).

\section{Event Selection}

All data presented in the following were collected between the years 1998 and 2000 when HERA collided positrons (electrons) of $E_{e}=27.5 \mathrm{GeV}$ with protons of $E_{p}=920 \mathrm{GeV}$.

The kinematic ranges and the integrated luminosity of each measurement are shown in Table 1. The DIS kinematic variables $Q^{2}$ and $y$ are reconstructed from the scattered positron (electron).

\begin{tabular}{|c|c|c|c|c|}
\hline Measurement & $Q^{2}$-range & $y$-range & Luminosity $\left[\mathrm{pb}^{-1}\right]$ & $D^{*}$ yield \\
\hline ZEUS (DIS) & $1.5<Q^{2}<200 \mathrm{GeV}^{2}$ & $0.02<y<0.7$ & 82 & $253 \pm 21$ \\
\hline H1 (DIS) & $2<Q^{2}<200 \mathrm{GeV}^{2}$ & $0.05<y<0.7$ & 42.6 & $140 \pm 16$ \\
\hline ZEUS (PhP) & $Q^{2}<1 \mathrm{GeV}^{2}$ & $0.17<y<0.9$ & 78.6 & $454 \pm 30$ \\
\hline
\end{tabular}

Table 1: Kinematic range, integrated luminosity and diffractive $D^{*}$ yield of each measurement in DIS and photoproduction $(P h P)$ presented in this paper.

Photoproduction events are identified by the absence of a scattered positron (electron) in the ZEUS calorimeters. In this way the photon virtuality is restricted to $Q^{2}<1 \mathrm{GeV}^{2}$. The center-ofmass energy of the $\gamma p$-system $W$ which is correlated to the inelasticity $y$ of the scattered positron (electron) via $W=\sqrt{s \cdot y-Q^{2}} 1$ is calculated from the hadronic final state using combined information from the tracking detectors and the calorimeters. The kinematic restriction of the measurement in $y$ is given in Table 1 .

Diffractive events are selected by the absence of hadronic activity above noise thresholds in the forward regions of the experiments. By these selections a large rapidity gap of at least 2 (4) units for ZEUS (H1) is ensured between the systems $X$ and $Y$. For the ZEUS measurements the diffractive cross section is given for elastically scattered protons, whereas the $\mathrm{H} 1$ measurement includes a fraction of proton dissociative events with $M_{Y}<1.6 \mathrm{GeV}$. The difference between these cross section definitions has been estimated to be $10 \%$ [7]. The diffractive quantity $\mathrm{x}_{\mathbf{P}}$ is reconstructed as

$$
\mathrm{x}_{\mathbf{P}}=\frac{\mathrm{Q}^{2}+\mathrm{M}_{\mathrm{X}}^{2}}{\mathrm{Q}^{2}+\mathrm{W}^{2}}
$$

For the photoproduction measurement $Q^{2}$ in equation 2.1 is assumed to be zero. In addition for the $\mathrm{H} 1$ measurement an estimate for the fractional momentum of the diffractive exchange carried by the guon is given by

$$
\mathrm{z}_{\mathbf{P}}=\frac{\mathrm{Q}^{2}+\hat{\mathrm{s}}}{\mathrm{x}_{\mathbf{P}} \cdot \mathrm{y} \cdot \mathrm{s}}
$$

where $\hat{s}$ denotes the square of the invariant mass of the cic-pair, which is estimated from the reconstructed $D^{*}$-meson and the scattered positron.

\footnotetext{
${ }^{1}$ where $s$ indicates the squared centere-of-mass energy of the $e p$-system.
} 
The diffractive kinematic ranges in DIS are $\mathrm{x}_{\mathbf{P}}<0.035$ and $\beta<0.8$ for ZEUS and $\mathrm{x}_{\mathbf{P}}<0.04$, $M_{Y}<1.6 \mathrm{GeV}$ and $|t|<1 \mathrm{GeV}^{2}$ for $\mathrm{H} 1$. In photoproduction the diffractive kinematic range is given by $0.001<\mathrm{x}_{\mathbf{P}}<0.035$ and $|t|<1 \mathrm{GeV}^{2}$.

\section{3. $D^{*}$-Selection}

Open-charm events are tagged by $D^{* \pm}$-mesons, which are reconstructed from the decay $D^{*} \rightarrow$ $D^{0} \pi_{\text {slow }} \rightarrow K \pi \pi_{\text {slow. }} D^{*}$ candidates are restricted to a minimal $p_{t}$ of $1.5 \mathrm{GeV}(2 \mathrm{GeV})$ in the ZEUS (H1) analysis and are required to be well contained within the acceptance of the tracking detectors. The final number of $D^{*}$-mesons is obtained by the $\Delta M=\left(M\left(K, \pi, \pi_{\text {slow }}\right)-M(K, \pi)\right)$ method [8], where $M\left(K, \pi, \pi_{\text {slow }}\right)$ denotes the invariant mass of the $K, \pi$ and the $\pi_{\text {slow }}$ candidate and $M(K, \pi)$ denotes the invariant mass of the $K$ and the $\pi$ candidate only. The $\Delta M$-distributions in DIS and photoproduction are shown in Fig. $2 \mathrm{a}$ and b. For the $\mathrm{H} 1$ measurement the number of $D^{*}$-mesons is obtained from a fit to the $\Delta M$ distribution, for the ZEUS measurements it is obtained by subtracting a 'wrong-charge'- $D^{*}$ sample where the decay products of the $D^{0}$-meson are chosen to be likesign to estimate the combinatorial background. The numbers of diffractive $D^{*}$-mesons for each measurement are shown in Table 1.

\section{Comparison with Calculations in LO and NLO}

The DIS measurements are compared to NLO calculations [9] [10]. For the H1 measurement DPDFs are used, which were extracted from a DGLAP NLO QCD fit to the reduced diffractive cross section of inclusive scattering at $\mathrm{H} 1$ [3] [4]. The factorization and the renormalization scale are set to $\sqrt{Q^{2}+p_{t}^{2}+4 m_{c}^{2}}$ and the charm mass is assumed to be $m_{c}=1.5 \mathrm{GeV}$. For the ZEUS measurement several NLO DPDFs from fits to combined H1 and ZEUS inclusive diffractive measurements are used [11]. The factorization and the renormalization scale are set to $\sqrt{Q^{2}+4 m_{c}^{2}}$ and the charm mass is assumed to be $m_{c}=1.45 \mathrm{GeV}$. In photoproduction the data are compared to the predictions of the leading-order (LO) Monte Carlo generator RAPGAP [12], where parton showers are included into the calculations to simulate higher order QCD effects. The calculations are based on LO DPDFs extracted from inclusive diffractive scattering at $\mathrm{H} 1$ [3].

\section{Results}

In Fig. 4 the measured cross sections from H1 (Fig. 4 a-c) and ZEUS (Fig. $4 \mathrm{c}$ and d) are compared to the corresponding NLO calculations as a function of $\mathrm{x}_{\mathbf{P}}(\mathrm{a}), \mathrm{z}_{\mathbf{P}}(\mathrm{b}), p_{t}\left(D^{*}\right)$ (c) and $\eta\left(D^{*}\right)$ (d). For the $\mathrm{H} 1$ measurement the indicated (red) error bands correspond to a variation of the renormalization and the factorization scale by a factor of 2 and a variation of the charm mass in the range of $m_{c}=1.35 \ldots 1.65 \mathrm{GeV}$. For the ZEUS measurement the (yellow) error band corresponds to a variation of the charm mass in the range of $m_{c}=1.3 \ldots 1.6 \mathrm{GeV}$. In Fig. $4 \mathrm{c}$ the measurements of ZEUS and $\mathrm{H} 1$ are shown in the overlapping kinematic region. For this comparison the ZEUS datapoints have been extrapolated to the $\mathrm{H} 1$ kinematic range. The agreement between the two experiments and between the measurements and the NLO calculations (especially with the more 
recent H1 DPDFs) is very good, which indicates that QCD factorization for diffractive open-charm production in DIS is fulfilled.

In Fig. 5 the open-charm contribution to the diffractive proton structure function $F_{2}^{D(3)}$ as measured by the ZEUS collaboration is compared to several predictions based on a set of DPDFs extracted by [11]. The indicated (yellow) error band corresponds to a variation of the charm mass in the range of $m_{c}=1.3 \ldots 1.6 \mathrm{GeV}$. A high sensitivity to the choice of DPDFs in general and a good agreement with the standard choice of DPDFs is observed.

In Fig. 3 the measured cross section of diffractive photoproduction of $D^{*}$-mesons is compared to the predictions from RAPGAP. A good agreement in shape is observed but the Monte Carlo prediction overestimates the over all normalization of the data roughly by a factor of three ${ }^{2}$.

\section{Conclusions}

Recent measurements of diffractive open-charm production in DIS and photoproduction at HERA were presented. The charm quark is tagged by the reconstruction of $D^{*}$-mesons. In DIS a good agreement with predictions from different NLO DPDFs is observed by H1 and ZEUS. The measured cross sections of both experiments are in good agreement in the region of overlap. Especially the comparisons with the more recent H1 DPDFs indicate that QCD factorization for diffractive open-charm production in DIS is fulfilled. A preliminary measurement in photoproduction shows a good agreement in shape with the predictions of the LO Monte Carlo generator RAPGAP, but the prediction overestimates the over all normalization of the data roughly by a factor of three.

\section{References}

[1] J. Collins, Phys. Rev. D 57 (1998) 3051 and erratum ibid. D61 (2000) 019902.

[2] V. Gribov, L. Lipatov, Sov. J. Nucl. Phys 15 (1972) 438 and 675.

Y. Dokshitzer, Sov. Phys. JETP 45 (1977) 641.

G. Altarelli, G. Parisi, Nucl. Phys. B 126 (1977) 298.

[3] H1 Collaboration, C. Adloff et al., Z. Phys. C 76 (1997) 613.

[4] H1 Collaboration, paper 980 submitted to 31. Intl. Conf. on High Energy Physics, ICHEP 2002, Amsterdam.

[5] ZEUS Collaboration, M. Derrick et al., Nucl. Phys. B 672 (2003) 3-35.

[6] ZEUS Collaboration, paper 268 submitted to 22. Intl. Symp. on Lepton-Photon Interactions at High Energy, 2005, Uppsala.

[7] H1 Collaboration, paper 178 submitted to 32. Intl. Conf. on High Energy Physics, ICHEP, 2004, Beijing.

[8] G. J. Feldman et al., Phys. Rev. Lett. 38, 1977 (1313).

[9] B. W. Harris, J. Smith, Nucl.Phys. B 452 (1995) 109, hep-ph/9503484.

\footnotetext{
${ }^{2}$ In a more recent comparison to a NLO calculation based on the H1 DPDFs however, a very good agreement with the data in shape and normalization has been observed [13].
} 
[10] L. Alvero, J. C. Collins, J. J. Whitmore, PSU-HT 200 (1998) 13, hep-ph/9806340.

[11] L. Alvero et al., Phys.Rev. D 59, 074022 (1999).

[12] H. Jung, Comp. Phys. Comm. 86 (1995) 147.

[13] I. Melzer, Conference Contribution, Intl. Conf. on the Structure and Interactions of the Photon 2005 (Session VIa).

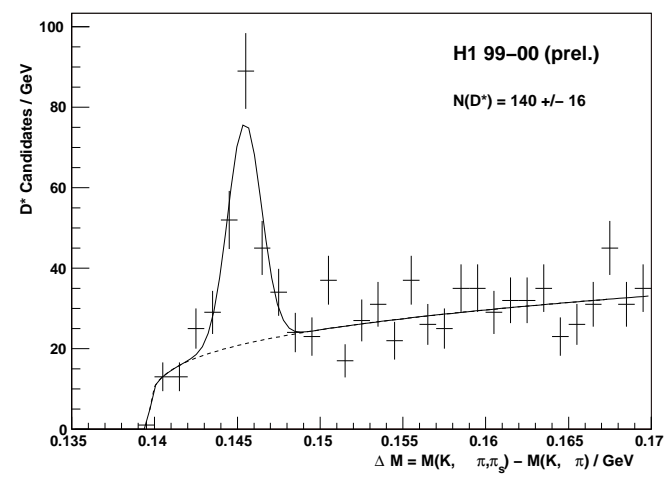

a)

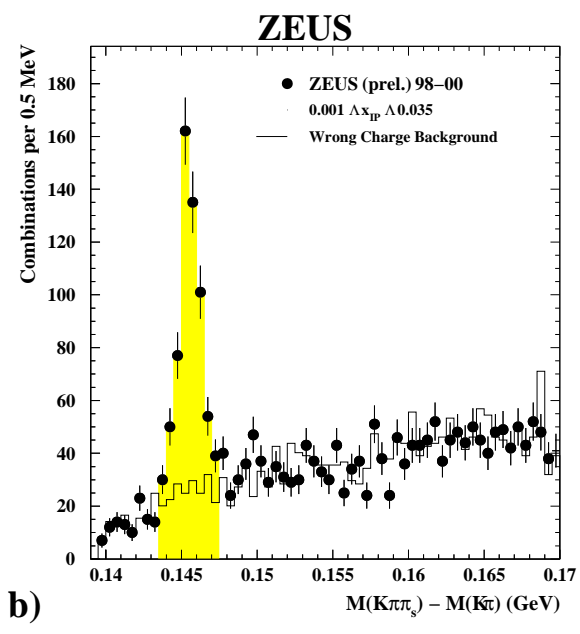

b)

Figure 2: Event yields for diffractive $D^{*}$-production in DIS at HI (a) and in photoproduction at ZEUS (b). Shown is the $\Delta M$-distribution after each final event selection.
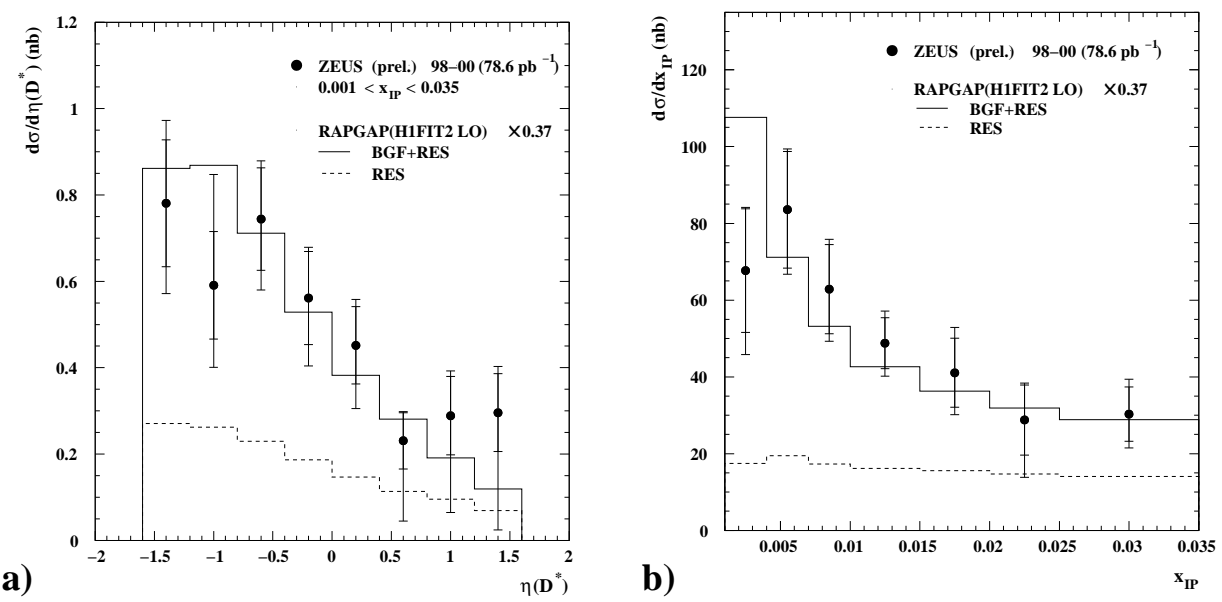

Figure 3: Photoproduction of diffractive $D^{*}$-mesons. Shown is the production cross section as a function of $\eta\left(D^{*}\right)(a)$ and $\mathrm{x}_{\mathbf{P}}(b)$ compared to the prediction of the LO Monte Carlo RAPGAP based on LO DPDFs obtained by $\mathrm{Hl}$ [3]. The Monte Carlo prediction is scaled by a factor of 0.37. 

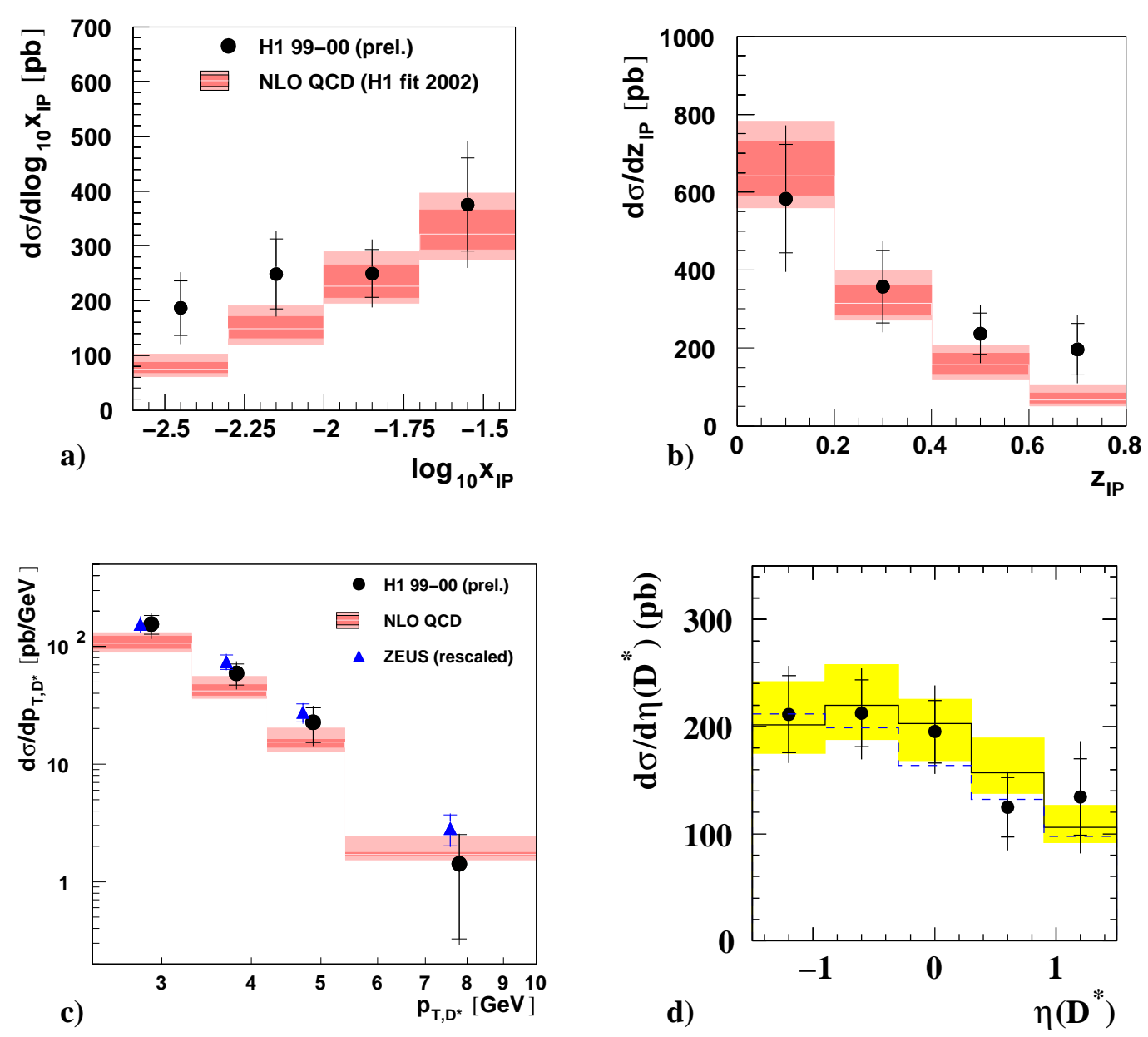

Figure 4: Diffractive $D^{*}$-meson production in DIS measured by $H 1$ (a-c) and ZEUS (c and d). Shown is the production cross section as a function of $\mathrm{x}_{\mathbf{P}}(a), \mathrm{z}_{\mathbf{P}}(b), p_{t}\left(D^{*}\right)(c)$ and $\eta\left(D^{*}\right)(d)$. Figure (c) shows a comparison between the ZEUS and the HI measurement in the overlapping kinematic range. For the comparison the ZEUS datapoints have been extrapolated to the H1 kinematic range. The meaning of the indicated bands is described in the text. 


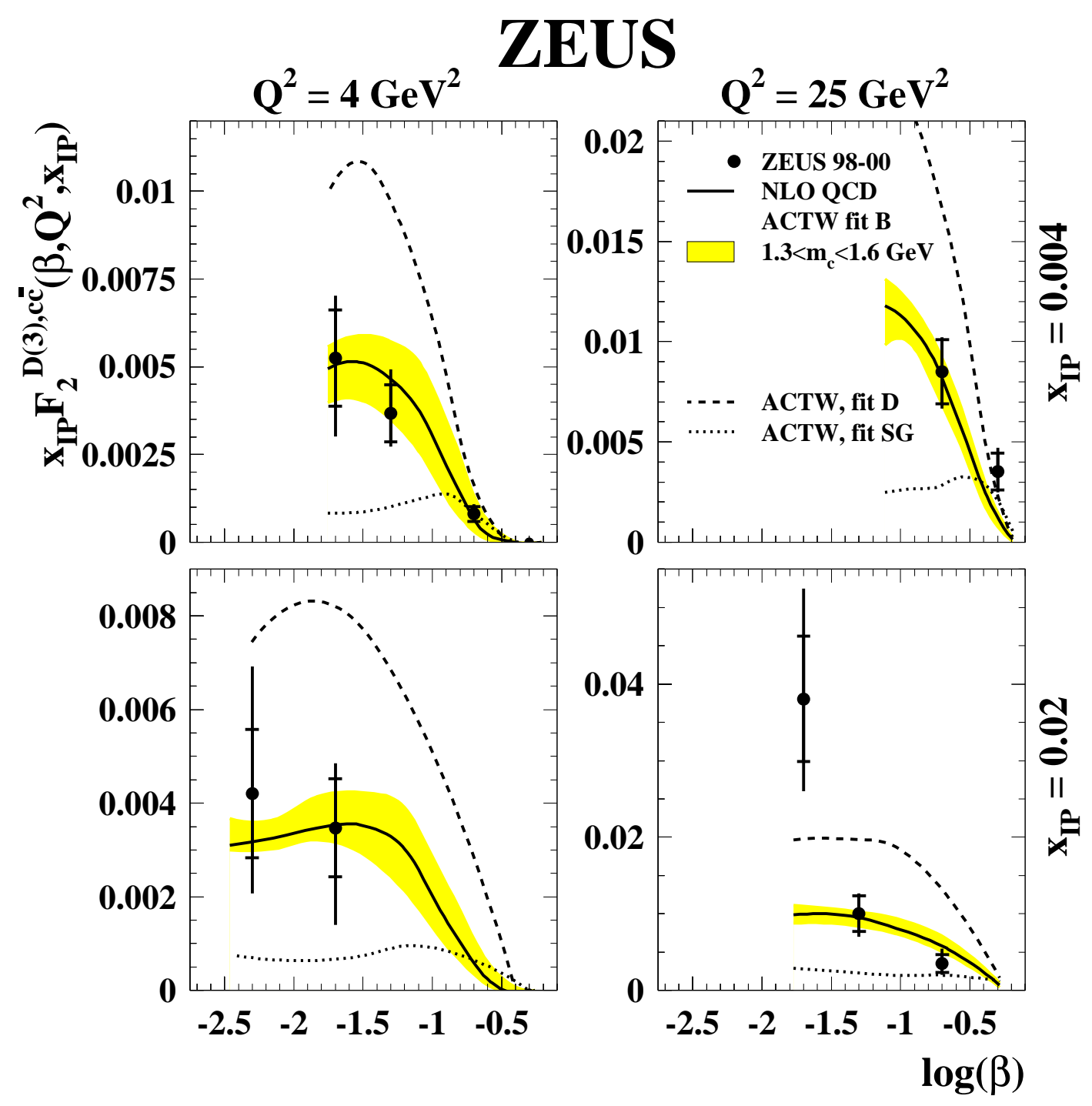

Figure 5: ZEUS measurement of the open-charm contribution to the diffractive proton structure function $F_{2}^{D(3)}$. The measurement is compared to several NLO predictions based on a selection of DPDFs [11]. The meaning of the indicated bands is described in the text. 